\title{
A SL(2) COVARIANT THEORY OF GENUS 2 HYPERELLIPTIC FUNCTIONS
}

\author{
CHRIS ATHORNE, J. C. EILBECK, AND V. Z. ENOLSKII
}

\begin{abstract}
We present an algebraic formulation of genus 2 hyperelliptic functions which exploits the underlying covariance of the family of genus 2 curves. This allows a simple interpretation of all identities in representation theoretic terms. We show how the classical theory is recovered when one branch point is moved to infinity.
\end{abstract}

\section{INTRODUCTION}

We will consider the family of curves in $\mathbb{C}^{2}$

$$
y^{2}=\lambda_{6} x^{6}+\lambda_{5} x^{5}+\lambda_{4} x^{4}+\lambda_{3} x^{3}+\lambda_{2} x^{2}+\lambda_{1} x+\lambda_{0},
$$

where the $\lambda_{i}$ are complex parameters. The generic member of this family is a curve of genus 2 with branch points in general position. In the classical theory one branch point is usually moved to infinity using a rational linear transformation so that $\lambda_{6}=0$. The value of $\lambda_{5}$ is simultaneously taken to equal 4 .

The problem of uniformization $[4,8]$ of the Jacobian $S y m^{2} \mathcal{V}$ of any generic curve, $\mathcal{V}$, of this family is solved, after repositioning the branch point, by defining three two index functions on the Jacobian. These functions are rational in positions $\left(x_{1}, y_{1}\right)$ and $\left(x_{2}, y_{2}\right)$ on the curve:

$$
\begin{aligned}
& \wp_{22}=x_{1}+x_{2}, \\
& \wp_{12}=-x_{1} x_{2}, \\
& \wp_{11}=\frac{F^{0}\left(x_{1}, x_{2}\right)-2 y_{1} y_{2}}{4\left(x_{1}-x_{2}\right)^{2}},
\end{aligned}
$$

where $F^{0}\left(x_{1}, x_{2}\right)$ is the polar form

$$
\begin{gathered}
F^{0}\left(x_{1}, x_{2}\right)=4\left(x_{1} x_{2}\right)^{2}\left(x_{1}+x_{2}\right)+2 \lambda_{4}\left(x_{1} x_{2}\right)^{2}+\lambda_{3}\left(x_{1} x_{2}\right)\left(x_{1}+x_{2}\right) \\
+2 \lambda_{2}\left(x_{1} x_{2}\right)+\lambda_{1}\left(x_{1}+x_{2}\right)+2 \lambda_{0} .
\end{gathered}
$$

The three $\wp$ functions are meromorphic functions of variables $u_{1}$ and $u_{2}$, on the Jacobi variety, defined by holomorphic differentials

$$
\begin{aligned}
& d u_{1}=\frac{d x_{1}}{y_{1}}+\frac{d x_{2}}{y_{2}}, \\
& d u_{2}=\frac{x_{1} d x_{1}}{y_{1}}+\frac{x_{2} d x_{2}}{y_{2}} .
\end{aligned}
$$

Date: July 29, 2002.

1991 Mathematics Subject Classification. Primary 14K05; Secondary 14H70,11F70. 
As we shall need them frequently we insert here the expressions for the corresponding derivatives in terms of the $x_{i}$ and $y_{i}$

$$
\begin{aligned}
\frac{\partial}{\partial u_{1}} & =\frac{1}{x_{1}-x_{2}}\left(-x_{2} y_{1} \frac{\partial}{\partial x_{1}}+x_{1} y_{2} \frac{\partial}{\partial x_{2}}\right), \\
\frac{\partial}{\partial u_{2}} & =\frac{1}{x_{1}-x_{2}}\left(y_{1} \frac{\partial}{\partial x_{1}}-y_{2} \frac{\partial}{\partial x_{2}}\right) .
\end{aligned}
$$

With respect to these variables the $\wp$ functions satisfy exactness conditions

$$
\frac{\partial \wp_{12}}{\partial u_{1}}=\frac{\partial \wp_{11}}{\partial u_{2}}, \quad \frac{\partial \wp_{22}}{\partial u_{1}}=\frac{\partial \wp_{12}}{\partial u_{2}},
$$

and can be written as double logarithmic derivatives of an entire function, $\sigma$, on the Jacobi variety

$$
\wp_{r s}=-\frac{\partial^{2} \ln \sigma\left(u_{1}, u_{2}\right)}{\partial u_{r} \partial u_{s}} .
$$

The three index symbols $\wp_{i j k}$, defined by differentiation with respect to the $u_{i}$, are also rational functions in the $x_{i}$ and $y_{i}$. In fact

$$
\begin{aligned}
& y_{1}=x_{1} \wp_{222}+\wp_{122}, \\
& y_{2}=x_{2} \wp_{222}+\wp_{122},
\end{aligned}
$$

and so the points $\left(\left(x_{1}, y_{1}\right),\left(x_{2}, y_{2}\right)\right)$ of $S y m^{2} \mathcal{V}$ can be expressed as rational functions in the two and three index $\wp$ symbols. This is the solution to the uniformization problem up to the study of the properties of the $\wp$ functions themselves from the identities and differential equations they satisfy.

One important identity for the two index symbols is obtained by elimination of the $x_{i}$ from the defining equations and represents a realization of the Kummer surface, det $\mathbb{K}=0$, where:

$$
\mathbb{K}=\left(\begin{array}{cccc}
-\lambda_{0} & \frac{1}{2} \lambda_{1} & 2 \wp_{11} & -2 \wp_{12} \\
\frac{1}{2} \lambda_{1} & -\left(\lambda_{2}+4 \wp_{11}\right) & \frac{1}{2} \lambda_{3}+2 \wp_{12} & 2 \wp_{22} \\
2 \wp_{11} & \frac{1}{2} \lambda_{3}+2 \wp_{12} & -\left(\lambda_{4}+4 \wp_{22}\right) & 2 \\
-2 \wp_{12} & 2 \wp_{22} & 2 & 0
\end{array}\right)
$$

Formulae exist for products of three index symbols as cubics in the two index symbols (see Appendix E) and the final, classical identity amounts to a set of five partial differential equations for the $\wp$ functions, i.e. fourth order equations for the $\sigma$ function:

$$
\begin{aligned}
\wp_{2222}-6 \wp_{22}^{2} & =\frac{1}{2} \lambda_{3}+\lambda_{4} \wp_{22}+4 \wp_{12}, \\
\wp_{1222}-6 \wp_{22} \wp_{12} & =\lambda_{4} \wp_{12}-2 \wp_{11}, \\
\wp_{1122}-2 \wp_{22} \wp_{11}-4 \wp_{12}^{2} & =\frac{1}{2} \lambda_{3} \wp_{12}, \\
\wp_{1112}-6 \wp_{12} \wp_{11} & =-\lambda_{0}-\frac{1}{2} \lambda_{1} \wp_{22}+\lambda_{2} \wp_{12}, \\
\wp_{1111}-6 \wp_{11}^{2} & =-\frac{1}{2} \lambda_{0} \lambda_{4}+\frac{1}{8} \lambda_{1} \lambda_{3}-3 \lambda_{0} \wp_{22}+\lambda_{1} \wp_{12}+\lambda_{2} \wp_{11} .
\end{aligned}
$$

The purpose of the current work is to consider the role of representations of $S L_{2}(\mathbb{C})$ in the above uniformization theory. Although there are tantalising glimpses in the classical theory of objects which seem to want such an interpretation, these 
are obscured by the canonical decision to place one branch point at infinity, a decision which is not respected by the full $S L_{2}(\mathbb{C})$ action. In order to make the covariant nature of the theory apparent it is desirable firstly to retain general values for the $\lambda_{i}$, and secondly to define the $\wp$ functions appropriately. We indicate in the next section how our approach differs from the classical way of expressing covariance of which a more detailed exposition will be presented elsewhere [3].

We shall denote our new $\wp$ functions simply by the calligraphic symbol $\mathcal{P}$, thus: $\mathcal{P}_{i j}, \mathcal{P}_{i j k}, \mathcal{P}_{i j k \ldots l}$.

\section{A COVARIANT APPROACH.}

For ease of exchange with the representation theory we rewrite $\lambda_{i}$ as $\left(\begin{array}{l}6 \\ i\end{array}\right) g_{i}$. The curve

$$
y^{2}=g_{6} x^{6}+6 g_{5} x^{5}+15 g_{4} x^{4}+20 g_{3} x^{3}+15 g_{2} x^{2}+6 g_{1} x+g_{0},
$$

is transformed under the rational, $\mathrm{PSL}_{2}(\mathbb{C})$ map

$$
x \mapsto X=\frac{\alpha x+\beta}{\gamma x+\delta}, \quad y \mapsto Y=\frac{y}{(\gamma x+\delta)^{3}},
$$

to one of the same form but with new values for the parameters:

$$
Y^{2}=G_{6} X^{6}+6 G_{5} X^{5}+15 G_{4} X^{4}+20 G_{3} X^{3}+15 G_{2} X^{2}+6 G_{1} X+G_{0} .
$$

The parameters $\left(g_{6}, . ., g_{0}\right)$ transform to $\left(G_{6}, . ., G_{0}\right)$ under a seven dimensional, irreducible representation of $S L_{2}(\mathbb{C})$. The action on $x, y$ and the $g_{i}$ is generated by the vector fields $\left(\mathfrak{s l} l_{2}(\mathbb{C})\right)$,

$$
\begin{aligned}
& e=-\frac{\partial}{\partial x}+\sum_{0}^{6}(6-i) g_{i+1} \frac{\partial}{\partial g_{i}} \\
& f=x^{2} \frac{\partial}{\partial x}+3 x y \frac{\partial}{\partial y}+\sum_{0}^{6} i g_{i-1} \frac{\partial}{\partial g_{i}}, \\
& h=-2 x \frac{\partial}{\partial x}-3 y \frac{\partial}{\partial y}+\sum_{0}^{6}(2 i-6) g_{i} \frac{\partial}{\partial g_{i}},
\end{aligned}
$$

which are sections of the tangent bundle of a nine dimensional manifold with local coordinates $x, y, g_{0}, \ldots, g_{6}$.

Writing the curve as $y^{2}-g(x)=0$ one sees that it is a covariant for the $S L_{2}(\mathbb{C})$ action:

$$
\begin{aligned}
& e\left(y^{2}-g(x)\right)=0, \\
& f\left(y^{2}-g(x)\right)=6 x\left(y^{2}-g(x)\right), \\
& h\left(y^{2}-g(x)\right)=-6\left(y^{2}-g(x)\right) .
\end{aligned}
$$

This action induces an action on the holomorphic differentials $d u_{1}$ and $d u_{2}$ $(1.6,1.7)$

$$
\begin{aligned}
& d u_{1}=\frac{d x_{1}}{y_{1}}+\frac{d x_{2}}{y_{2}} \\
& d u_{2}=\frac{x_{1} d x_{1}}{y_{1}}+\frac{x_{2} d x_{2}}{y_{2}}
\end{aligned}
$$


namely:

$$
\left(\begin{array}{c}
d u_{1} \\
d u_{2}
\end{array}\right) \mapsto\left(\begin{array}{c}
d U_{1} \\
d U_{2}
\end{array}\right)=\left(\begin{array}{cc}
\delta & \gamma \\
\beta & \alpha
\end{array}\right)\left(\begin{array}{c}
d u_{1} \\
d u_{2}
\end{array}\right) .
$$

Accordingly,

$$
\left(\begin{array}{c}
\partial_{u_{1}} \\
\partial_{u_{2}}
\end{array}\right) \mapsto\left(\begin{array}{c}
\partial_{U_{1}} \\
\partial_{U_{2}}
\end{array}\right)=\left(\begin{array}{cc}
\alpha & -\beta \\
-\gamma & \delta
\end{array}\right)\left(\begin{array}{c}
\partial_{u_{1}} \\
\partial_{u_{2}}
\end{array}\right) .
$$

and consequently the second derivatives with respect to $u_{1}$ and $u_{2}$ transform according to the three dimensional symmetric product of the two dimensional representation. Double index objects in the theory, such as $\wp_{i j}$, which are second derivatives should transform accordingly. But $\wp_{11}, \wp_{12}$ and $\wp_{22}$ do not do so if they are taken to be defined by equations $(1.2,1.4)$ for the generic curve, $F^{0}$ being replaced the corresponding generic polar form.

Instead, in the standard treatments [5] the $\wp_{i j}$ are defined for branch points in general position by linear sums of the above $\wp_{i j}$ and the addition of certain constants $[5,3]$ in such a way as to force the correct transformation properties. These generalised functions (given the same labels) are then seen to satisfy the five equations,

$$
\begin{gathered}
-\frac{1}{3}\left(\wp_{2222}-6 \wp_{22}^{2}\right)=g_{2} g_{6}-4 g_{3} g_{5}+3 g_{4}^{2}+g_{4} \wp_{22}-2 g_{5} \wp_{12}+g_{6} \wp_{11}, \\
-\frac{1}{3}\left(\wp_{1222}-6 \wp_{22} \wp_{12}\right)=\frac{1}{2}\left(g_{1} g_{6}-3 g_{2} g_{5}+2 g_{3} g_{4}\right) \\
+g_{3} \wp_{22}-2 g_{4} \wp_{12}+g_{5} \wp_{11}, \\
-\frac{1}{3}\left(\wp_{1122}-2 \wp_{22} \wp_{11}-4 \wp_{12}^{2}\right)=\frac{1}{6}\left(g_{0} g_{6}-9 g_{2} g_{4}+8 g_{3}^{2}\right) \\
+g_{2} \wp_{22}-2 g_{3} \wp_{12}+g_{4} \wp_{11}, \\
-\frac{1}{3}\left(\wp_{1112}-6 \wp_{12} \wp_{11}\right)=\frac{1}{2}\left(g_{0} g_{5}-3 g_{1} g_{4}+2 g_{2} g_{3}\right) \\
+g_{1} \wp_{22}-2 g_{2} \wp_{12}+g_{3} \wp_{11}, \\
-\frac{1}{3}\left(\wp_{1111}-6 \wp_{11}^{2}\right)=g_{0} g_{4}-4 g_{1} g_{3}+3 g_{2}^{2}+g_{0} \wp_{22}-2 g_{1} \wp_{12}+g_{2} \wp_{11} .
\end{gathered}
$$

which will be seen to be covariant (Section 4).

The drawback of the defining expressions for the generic curve is that covariance is forced by an unnatural choice of the generalised functions. The coefficients of their linear, defining expressions are functions of the coefficients of the transformation which moves a branch point to infinity and hence are functions of the $\lambda_{i}$.

In view of this we proceed to a natural, ab initio definition for the two index symbols associated with the generic curve which ensures their covariance. The price of such a covariant definition will be a slightly more complicated set of relations between indexed objects although these relations will have the virtues both of being generated by simple algebraic operations and of being classified by the representations of $S L_{2}(\mathbb{C})$ they carry.

One checks that

$$
\mathcal{P}_{22}=\frac{2}{x_{1}-x_{2}}, \quad \mathcal{P}_{12}=-\frac{x_{1}+x_{2}}{x_{1}-x_{2}}, \quad \mathcal{P}_{11}=\frac{2 x_{1} x_{2}}{x_{1}-x_{2}},
$$


have the following properties:

$$
\begin{aligned}
& \mathcal{P}_{22} x_{1} x_{2}+\mathcal{P}_{12}\left(x_{1}+x_{2}\right)+\mathcal{P}_{11}=x_{2}-x_{1}, \\
& \mathcal{P}_{22} x_{1}^{2}+2 \mathcal{P}_{12} x_{1}+\mathcal{P}_{11}=0 \\
& \mathcal{P}_{22} x_{2}^{2}+2 \mathcal{P}_{12} x_{2}+\mathcal{P}_{11}=0 \\
& \frac{\partial}{\partial u_{1}} \mathcal{P}_{22}=\frac{\partial}{\partial u_{2}} \mathcal{P}_{12}, \quad \frac{\partial}{\partial u_{1}} \mathcal{P}_{12}=\frac{\partial}{\partial u_{2}} \mathcal{P}_{11}, \\
& \mathcal{P}_{12}^{2}-\mathcal{P}_{11} \mathcal{P}_{22}=1 .
\end{aligned}
$$

It follows from these properties that the triplet $\left(\mathcal{P}_{11}, \mathcal{P}_{12}, \mathcal{P}_{22}\right)$ transforms as does $\left(\partial_{u_{1}}^{2}, \partial_{u_{1}} \partial_{u_{2}}, \partial_{u_{2}}^{2}\right)$, that we may put $\mathcal{P}_{i j}=\partial_{u_{i}} \partial_{u_{j}} \Upsilon$ and that $\Upsilon$ satisfies the MongeAmpére equation,

$$
\left(\frac{\partial^{2} \Upsilon}{\partial u_{1} \partial u_{2}}\right)^{2}-\frac{\partial^{2} \Upsilon}{\partial u_{1}^{2}} \frac{\partial^{2} \Upsilon}{\partial u_{2}^{2}}=1
$$

so that $\Upsilon=\Upsilon\left(u_{1}, u_{2}\right)$ is a surface of constant negative curvature equal to -2 .

The expressions for the corresponding three index symbols, following from differentiation, are:

$$
\begin{aligned}
& \mathcal{P}_{111}=2 \frac{y_{1} x_{2}^{3}+y_{2} x_{1}^{3}}{\left(x_{1}-x_{2}\right)^{3}}, \\
& \mathcal{P}_{112}=-2 \frac{y_{1} x_{2}^{2}+y_{2} x_{1}^{2}}{\left(x_{1}-x_{2}\right)^{3}}, \\
& \mathcal{P}_{122}=2 \frac{y_{1} x_{2}+y_{2} x_{1}}{\left(x_{1}-x_{2}\right)^{3}}, \\
& \mathcal{P}_{222}=-2 \frac{y_{1}+y_{2}}{\left(x_{1}-x_{2}\right)^{3}} .
\end{aligned}
$$

One constructs symbols of arbitrary numbers of indices by differentiation with respect to $u_{1}$ and $u_{2}$. The symbol $\mathcal{P}_{\{\mathbf{p}, \mathbf{q}\}}$ will denote a $\mathcal{P}$ function having $p+q$ indices, $p$ of them equal to 1 and the remaining $q$ equal to 2 . So

$$
\begin{aligned}
& \partial_{u_{1}} \mathcal{P}_{\{\mathbf{p}, \mathbf{q}\}}=\mathcal{P}_{\{\mathbf{p}+\mathbf{1}, \mathbf{q}\}}, \\
& \partial_{u_{2}} \mathcal{P}_{\{\mathbf{p}, \mathbf{q}\}}=\mathcal{P}_{\{\mathbf{p}, \mathbf{q}+\mathbf{1}\}} .
\end{aligned}
$$

There are $p+q+1$ symbols with $p+q$ indices and they form a basis for a $p+q+1$ dimensional irreducible representation of $S L_{2}(\mathbb{C})$ induced by the action on $x$ and $y$ and generated by the action of $\mathfrak{s} l_{2}(\mathbb{C})$ given by:

$$
\begin{aligned}
& e\left(\mathcal{P}_{\{\mathbf{p}, \mathbf{q}\}}\right)=p \mathcal{P}_{\{\mathbf{p}-\mathbf{1}, \mathbf{q}+\mathbf{1}\}}, \\
& f\left(\mathcal{P}_{\{\mathbf{p}, \mathbf{q}\}}\right)=q \mathcal{P}_{\{\mathbf{p}+\mathbf{1}, \mathbf{q}-\mathbf{1}\}}, \\
& h\left(\mathcal{P}_{\{\mathbf{p}, \mathbf{q}\}}\right)=(p-q) \mathcal{P}_{\{\mathbf{p}, \mathbf{q}\}} .
\end{aligned}
$$

They are the highest weight irreducibles in the $N$ fold symmetric product of the fundamental representation.

The derivatives raise the dimension of a representation. They are not, however, $\mathfrak{s} l_{2}(\mathbb{C})$-module homomorphisms but satisfy the following important commutation relations:

$$
\left[e, \partial_{u_{1}}\right]=\partial_{u_{2}}, \quad\left[f, \partial_{u_{1}}\right]=0, \quad\left[h, \partial_{u_{1}}\right]=\partial_{u_{1}}
$$




$$
\left[e, \partial_{u_{2}}\right]=0, \quad\left[f, \partial_{u_{2}}\right]=\partial_{u_{1}}, \quad\left[h, \partial_{u_{2}}\right]=-\partial_{u_{2}} .
$$

In the simplest case, $\mathcal{P}_{\{\mathbf{0}, \mathbf{2}\}}=\mathcal{P}_{22}, \mathcal{P}_{\{\mathbf{1}, \mathbf{1}\}}=\mathcal{P}_{12}$ and $\mathcal{P}_{\{\mathbf{2}, \mathbf{0}\}}=\mathcal{P}_{11}$, so that $e\left(\mathcal{P}_{11}\right)=2 \mathcal{P}_{12}, e\left(\mathcal{P}_{12}\right)=\mathcal{P}_{22}, e\left(\mathcal{P}_{22}\right)=0, f\left(\mathcal{P}_{11}\right)=0, f\left(\mathcal{P}_{12}\right)=\mathcal{P}_{11}$ and $f\left(\mathcal{P}_{22}\right)=$ $2 \mathcal{P}_{12}$. One easily checks that the condition $\mathcal{P}_{12}^{2}-\mathcal{P}_{11} \mathcal{P}_{22}=1$ is an invariant constraint:

$$
\begin{aligned}
& e\left(\mathcal{P}_{12}^{2}-\mathcal{P}_{11} \mathcal{P}_{22}\right)=2 \mathcal{P}_{12} \mathcal{P}_{22}-2 \mathcal{P}_{12} \mathcal{P}_{22}=0 \\
& f\left(\mathcal{P}_{12}^{2}-\mathcal{P}_{11} \mathcal{P}_{22}\right)=2 \mathcal{P}_{12} \mathcal{P}_{11}-2 \mathcal{P}_{11} \mathcal{P}_{12}=0 .
\end{aligned}
$$

From these we derive, by differentiation, a pair of identities involving two and three index objects,

$$
\begin{aligned}
& \mathcal{P}_{11} \mathcal{P}_{122}-2 \mathcal{P}_{12} \mathcal{P}_{112}+\mathcal{P}_{22} \mathcal{P}_{111}=0 \\
& \mathcal{P}_{11} \mathcal{P}_{222}-2 \mathcal{P}_{12} \mathcal{P}_{122}+\mathcal{P}_{22} \mathcal{P}_{112}=0
\end{aligned}
$$

where the left hand sides comprise an irreducible two dimensional representation of $S L_{2}(\mathbb{C})$.

A further differentiation yields an irreducible three dimensional representation of $S L_{2}(\mathbb{C})$ involving also 4 -index objects

$$
\begin{array}{r}
2 \mathcal{P}_{1112} \mathcal{P}_{12}+2 \mathcal{P}_{112}^{2}-\mathcal{P}_{1111} \mathcal{P}_{22}-2 \mathcal{P}_{111} \mathcal{P}_{122}-\mathcal{P}_{11} \mathcal{P}_{1122}=0 \\
2 \mathcal{P}_{1122} \mathcal{P}_{12}+\mathcal{P}_{112} \mathcal{P}_{122}-\mathcal{P}_{1112} \mathcal{P}_{22}-\mathcal{P}_{111} \mathcal{P}_{222}-\mathcal{P}_{11} \mathcal{P}_{1222}=0 \\
2 \mathcal{P}_{1222} \mathcal{P}_{12}+2 \mathcal{P}_{122}^{2}-\mathcal{P}_{1122} \mathcal{P}_{22}-2 \mathcal{P}_{112} \mathcal{P}_{222}-\mathcal{P}_{11} \mathcal{P}_{2222}=0
\end{array}
$$

We must require that all equations of the theory be invariant or, more generally, covariant in this way.

\section{IdENTIties}

Denote by $V_{n+1}$ the $n+1$ dimensional irreducible representation spanned by the symbols carrying $n$ indices and by $G_{7}$ the seven dimensional representation carried by the coefficients $\left\{g_{6}, g_{5}, g_{4}, g_{3}, g_{2}, g_{1}, g_{0}\right\}$ of the family of curves. When we write $A_{n}$ we will mean a generic $n$ dimensional representation.

An important construction for us is the following.

On the one hand note that $\partial_{u_{i}}$ are almost intertwining operators for the action of $S L_{2}(\mathbb{C})$ in that they commute with one of $f$ and $e$ and hence map highest or lowest weight vectors in one representation to highest or lowest weight vectors in another. This observation holds for tensor products of representations too, $\partial_{u_{i}}$ being extended, like $e$ and $f$, as a derivation. However, not all representations can be obtained by a chain of such almost intertwining operators. On the other hand, if we modify the action to the following Hirota maps,

$$
\begin{aligned}
D_{1} & : a \otimes b \mapsto \partial_{u_{1}} a \otimes b-a \otimes \partial_{u_{1}} b, \\
D_{2}: a \otimes b & \mapsto \partial_{u_{2}} a \otimes b-a \otimes \partial_{u_{2}} b, \\
D_{12}: a \otimes b & \mapsto \partial_{u_{1}} a \otimes \partial_{u_{2}} b-\partial_{u_{2}} a \otimes \partial_{u_{1}} b,
\end{aligned}
$$

then all the highest and lowest weight vectors for irreducibles in the decomposition (plethysm) of an arbitrary tensor product can be constructed. More details are available in $[1,2]$ where, the reader should note, only one of these Hirota maps is used. 
It is interesting to note that the Monge-Ampére equation (2.21) has the form:

$$
\frac{1}{2} \operatorname{Sym}\left(D_{12}^{2} \Upsilon \otimes \Upsilon\right)+1=0
$$

Although this is an unconventional formulation, such "Hirota" forms are of enormous value and influence in the theory of integrable differential equations where they play a role in the classification of systems, in forming ansätze for their solutions and in their infinite dimensional geometric description. But it is amusing to note $[6,7]$ that Baker in his book on "Multiply Periodic Functions" of 1907 [5, p49] introduces the notation

$$
\Delta^{n} \sigma(x) \sigma\left(x^{\prime}\right)=\lim _{x \rightarrow x^{\prime}}\left(\partial_{x}-\partial_{x^{\prime}}\right)^{n} \sigma(x) \sigma\left(x^{\prime}\right),
$$

which is precisely the conventional Hirota operator and writes the left hand sides of equations (2.14) in a bilinear "Hirota" form. In view of this it seems appropriate to christen the generalised maps we have introduced above, Baker-Hirota maps.

We now take a systematic look at some simple tensor products.

3.1. $\operatorname{Sym}\left(V_{3} \otimes V_{3}\right)$. The tensor product $\operatorname{Sym}\left(V_{3} \otimes V_{3}\right)$ decomposes into irreducibles $A_{1} \oplus A_{5}$ of dimensions one and five. The first is just the invariant, $\mathcal{P}_{12}^{2}-\mathcal{P}_{11} \mathcal{P}_{22}$. The $A_{5}$ is the representation with basis $\left\{\mathcal{P}_{22}^{2}, \mathcal{P}_{12} \mathcal{P}_{22}, 2 \mathcal{P}_{12}^{2}+\mathcal{P}_{11} \mathcal{P}_{22}, \mathcal{P}_{12} \mathcal{P}_{11}, \mathcal{P}_{11}^{2}\right\}$.

3.2. $V_{4} \otimes V_{3}$. The tensor product $V_{4} \otimes V_{3}$ decomposes as $A_{2} \oplus A_{4} \oplus A_{6}$. The highest weight elements of each representation (by highest weight we will mean, merely as a matter of convention, "belonging to the kernel of $f$ "), can be found using Hirota maps. We indicate the highest weight element by writing it explicitly as an argument to $A_{n}$ :

$$
\begin{aligned}
& V_{4} \otimes V_{3}=A_{6}\left(\mathcal{P}_{\{\mathbf{3}, \mathbf{0}\}} \otimes \mathcal{P}_{\{\mathbf{2}, \mathbf{0}\}}\right) \\
& \oplus A_{4}\left(\mathcal{P}_{\{\mathbf{2}, \mathbf{1}\}} \otimes \mathcal{P}_{\{\mathbf{2}, \mathbf{0}\}}-\mathcal{P}_{\{\mathbf{3}, \mathbf{0}\}} \otimes \mathcal{P}_{\{\mathbf{1}, \mathbf{1}\}}\right) \\
& \oplus A_{2}\left(\mathcal{P}_{\{\mathbf{1}, \mathbf{2}\}} \otimes \mathcal{P}_{\{\mathbf{2}, \mathbf{0}\}}-2 \mathcal{P}_{\{\mathbf{2}, \mathbf{1}\}} \otimes \mathcal{P}_{\{\mathbf{1}, \mathbf{1}\}}+\mathcal{P}_{\{\mathbf{3}, \mathbf{0}\}} \otimes \mathcal{P}_{\{\mathbf{0}, \mathbf{2}\}}\right)
\end{aligned}
$$

The $A_{2}$ here is easily seen to be exactly the two dimensional representation (2.33).

The $A_{4}$ irreducible, expressed in terms of the original variables $\left(x_{i}, y_{i}\right)$, has the basis

$$
\begin{aligned}
\mathcal{P}_{111} \mathcal{P}_{12}-\mathcal{P}_{112} \mathcal{P}_{11} & =2 \frac{y_{1} x_{2}^{3}-y_{2} x_{1}^{3}}{\left(x_{1}-x_{2}\right)^{3}}, \\
\mathcal{P}_{112} \mathcal{P}_{12}-2 \mathcal{P}_{122} \mathcal{P}_{11}+\mathcal{P}_{111} \mathcal{P}_{22} & =-6 \frac{y_{1} x_{2}^{2}-y_{2} x_{1}^{2}}{\left(x_{1}-x_{2}\right)^{3}} \\
\mathcal{P}_{122} \mathcal{P}_{12}-2 \mathcal{P}_{112} \mathcal{P}_{22}+\mathcal{P}_{222} \mathcal{P}_{11} & =-6 \frac{y_{1} x_{2}-y_{2} x_{1}}{\left(x_{1}-x_{2}\right)^{3}} \\
\mathcal{P}_{222} \mathcal{P}_{12}-\mathcal{P}_{122} \mathcal{P}_{22} & =2 \frac{y_{1}-y_{2}}{\left(x_{1}-x_{2}\right)^{3}} .
\end{aligned}
$$

These expressions complement those for the three index symbols themselves. These, however, are symmetric under interchange of points $\left(x_{1}, y_{1}\right)$ and $\left(x_{2}, y_{2}\right)$ 
whereas the former are antisymmetric. In particular they allow us to recover expressions for $y_{1}$ and $y_{2}$ and not only their symmetric functions:

$$
\begin{aligned}
& x_{1}=\frac{1-\mathcal{P}_{12}}{\mathcal{P}_{22}}, \quad x_{2}=-\frac{1+\mathcal{P}_{12}}{\mathcal{P}_{22}} \\
& y_{1}=\frac{2}{\mathcal{P}_{22}} \partial_{u_{2}}\left(\frac{1-\mathcal{P}_{12}}{\mathcal{P}_{22}}\right), \quad y_{2}=\frac{2}{\mathcal{P}_{22}} \partial_{u_{2}}\left(\frac{1+\mathcal{P}_{12}}{\mathcal{P}_{22}}\right) .
\end{aligned}
$$

Because the $\mathcal{P}_{i j}$ are functions of two complex variables, these formulae do not constitute a unformization of the original genus two curve though they are a parametrization in a much weaker sense.

3.3. $\operatorname{Sym}\left(V_{4} \otimes V_{4}\right)$. The next interesting product is $\operatorname{Sym}\left(V_{4} \otimes V_{4}\right)$, the sum of an $A_{7}$ and an $A_{3}$.

$$
\begin{aligned}
\operatorname{Sym}\left(V_{4} \otimes V_{4}\right) & =A_{7}\left(\mathcal{P}_{\{\mathbf{3}, \mathbf{0}\}} \otimes \mathcal{P}_{\{\mathbf{3}, \mathbf{0}\}}\right) \\
& \oplus A_{3}\left(\mathcal{P}_{\{\mathbf{3}, \mathbf{0}\}} \otimes \mathcal{P}_{\{\mathbf{1}, \mathbf{2}\}}-2 \mathcal{P}_{\{\mathbf{2}, \mathbf{1}\}} \otimes \mathcal{P}_{\{\mathbf{2}, \mathbf{1}\}}+\mathcal{P}_{\{\mathbf{1}, \mathbf{2}\}} \otimes \mathcal{P}_{\{\mathbf{3}, \mathbf{0}\}}\right) .
\end{aligned}
$$

The $A_{3}$ here is essentially $V_{3}$ up to a factor: $\mathcal{I}=\frac{2 y_{1} y_{2}}{\left(x_{1}-x_{2}\right)^{3}}$ which is easily seen to be invariant by applying $e$ and $f$. Thus,

$$
\begin{aligned}
\mathcal{P}_{111} \mathcal{P}_{122}-\mathcal{P}_{112}^{2} & =\mathcal{I} \mathcal{P}_{11} \\
\mathcal{P}_{111} \mathcal{P}_{222}-\mathcal{P}_{122} \mathcal{P}_{112} & =2 \mathcal{I} \mathcal{P}_{12} \\
\mathcal{P}_{112} \mathcal{P}_{222}-\mathcal{P}_{122}^{2} & =\mathcal{I} \mathcal{P}_{22}
\end{aligned}
$$

We can construct an explicitly invariant expression for $\mathcal{I}$ which is the trivial representation sitting inside $V_{4} \otimes V_{4} \otimes V_{3}$ :

$$
\mathcal{I}=-\frac{1}{2}\left|\begin{array}{ccc}
\mathcal{P}_{111} & \mathcal{P}_{112} & \mathcal{P}_{122} \\
\mathcal{P}_{112} & \mathcal{P}_{122} & \mathcal{P}_{222} \\
\mathcal{P}_{11} & \mathcal{P}_{12} & \mathcal{P}_{22}
\end{array}\right|
$$

Alternatively there is clearly a relation between the square of $\mathcal{I}$ and a rather unmanageable sextic in the two index symbols, see A.

The $A_{7}$ gives us a set of identities between two and three index symbols. The highest weight vector is

$$
\mathcal{P}_{111}^{2}=4 \frac{y_{1}^{2} x_{2}^{6}+y_{2}^{2} x_{1}^{6}}{\left(x_{1}-x_{2}\right)^{6}}+4 \mathcal{I} \frac{x_{1}^{3} x_{2}^{3}}{\left(x_{1}-x_{2}\right)^{3}},
$$

of which the right hand side can be written as a sextic function of the $\mathcal{P}_{i j}$ involving the invariant $\mathcal{I}=\frac{2 y_{1} y_{2}}{\left(x_{1}-x_{2}\right)^{3}}$, and the lowest weight vector (in the kernel of $e$ ) is,

$$
\mathcal{P}_{222}^{2}=4 \frac{y_{1}^{2}+y_{2}^{2}}{\left(x_{1}-x_{2}\right)^{6}}+4 \mathcal{I} \frac{1}{\left(x_{1}-x_{2}\right)^{3}} .
$$

The recursive application of $e$ to the first or of $f$ to this second equality generates a sequence of seven identities of which the left hand sides are all quadratics in the three index symbols. Rather than interrupt the flow, we present only the lowest 
weight identity here and the whole set in Appendix B.

$$
\begin{aligned}
\mathcal{P}_{222}^{2}- & \frac{1}{2} \mathcal{I}_{22}^{3}=\frac{1}{8} g_{0} \mathcal{P}_{22}{ }^{6}-\frac{3}{4} g_{1} \mathcal{P}_{12} \mathcal{P}_{22}{ }^{5}-\frac{15}{8} g_{2}\left(\mathcal{P}_{11} \mathcal{P}_{22}-2 \mathcal{P}_{12}{ }^{2}\right) \mathcal{P}_{22}{ }^{4} \\
+ & \frac{5}{2} g_{3} \mathcal{P}_{12}\left(3 \mathcal{P}_{11} \mathcal{P}_{22}-4 \mathcal{P}_{12}{ }^{2}\right) \mathcal{P}_{22}{ }^{3}+\frac{15}{8} g_{4}\left(8 \mathcal{P}_{12}{ }^{4}-8 \mathcal{P}_{11} \mathcal{P}_{12}{ }^{2} \mathcal{P}_{22}\right. \\
+ & \left.\mathcal{P}_{11}{ }^{2} \mathcal{P}_{22}{ }^{2}\right) \mathcal{P}_{22}{ }^{2}-\frac{3}{4} g_{5} \mathcal{P}_{12}\left(16 \mathcal{P}_{12}{ }^{4}-20 \mathcal{P}_{11} \mathcal{P}_{12}{ }^{2} \mathcal{P}_{22}\right. \\
+ & \left.5 \mathcal{P}_{11}{ }^{2} \mathcal{P}_{22}{ }^{2}\right) \mathcal{P}_{22}-\frac{1}{8} g_{6}\left(\mathcal{P}_{11} \mathcal{P}_{22}-2 \mathcal{P}_{12}{ }^{2}\right)\left(\mathcal{P}_{11}{ }^{2} \mathcal{P}_{22}{ }^{2}\right. \\
& \left.-16 \mathcal{P}_{11} \mathcal{P}_{12}{ }^{2} \mathcal{P}_{22}+16 \mathcal{P}_{12}{ }^{4}\right)
\end{aligned}
$$

Although these are the natural forms of the identities, it is possible to solve them for the binomials $\mathcal{P}_{i j k} \mathcal{P}_{l m n}$ in terms of $\mathcal{I}$ and the 2 -index functions. There are ten such binomials so one supplements the seven equations of the set using the three identities (3.14). The resulting system (in which $\mathcal{I}$ is regarded as a functional parameter) has rank 10 in the binomials and so can be solved. However the results are not particularly different from (3.20) to be worth displaying. $(3.20)$

It is interesting to note the following identity for the second term on the l.h.s. of

$$
\mathcal{I P}_{22}^{3}=-\left(\mathcal{P}_{222} \mathcal{P}_{12}+\mathcal{P}_{222}-\mathcal{P}_{122} \mathcal{P}_{22}\right)\left(\mathcal{P}_{222} \mathcal{P}_{12}-\mathcal{P}_{222}-\mathcal{P}_{122} \mathcal{P}_{22}\right)
$$

This can be shown by application of (2.33). Applying $f$ to the r.h.s. of this generates another 7-dimensional representation, the final equation factoring to give

$$
\mathcal{I} \mathcal{P}_{11}^{3}=-\left(\mathcal{P}_{111} \mathcal{P}_{12}-\mathcal{P}_{11} \mathcal{P}_{112}+\mathcal{P}_{111}\right)\left(\mathcal{P}_{111} \mathcal{P}_{12}-\mathcal{P}_{11} \mathcal{P}_{112}-\mathcal{P}_{111}\right)
$$

Corresponding to Baker's five equations for the four index objects, $\wp_{i j k l}$, we should like to find a set of five equations for the $\mathcal{P}_{i j k l}$. The tensor product $V_{5} \otimes$ $V_{3}$ decomposes as $A_{7} \oplus A_{5} \oplus A_{3}$ so it is natural to look at the five dimensional representation here. The lowest weight vector is

$$
\mathcal{P}_{2222} \mathcal{P}_{12}-\mathcal{P}_{1222} \mathcal{P}_{22}=-6 \frac{\left(y_{1}+y_{2}\right)\left(y_{1}-y_{2}\right)}{\left(x_{1}-x_{2}\right)^{5}}+\frac{\frac{d}{d x_{1}}\left(y_{1}\right)^{2}+\frac{d}{d x_{2}}\left(y_{2}\right)^{2}}{\left(x_{1}-x_{2}\right)^{4}}
$$

This is also obtained by differentiation of $\mathcal{P}_{222} \mathcal{P}_{12}-\mathcal{P}_{122} \mathcal{P}_{22}$ with respect to $u_{2}$. The right hand side can be calculated in a straightforward way to give the first term in the five dimensional representation

$$
\begin{array}{r}
\mathcal{P}_{2222} \mathcal{P}_{12}-\mathcal{P}_{1222} \mathcal{P}_{22}=-\frac{3}{2} g_{1} \mathcal{P}_{22}^{4}+\frac{15}{2} g_{2} \mathcal{P}_{12} \mathcal{P}_{22}^{3}-15 g_{3} \mathcal{P}_{12}^{2} \mathcal{P}_{22}^{2}+15 g_{4} \mathcal{P}_{12}^{3} \mathcal{P}_{22} \\
-\frac{3}{2} g_{5}\left(5 \mathcal{P}_{11}^{2} \mathcal{P}_{22}^{2}+4+10 \mathcal{P}_{11} \mathcal{P}_{22}\right)+\frac{3}{2} g_{6} \mathcal{P}_{11} \mathcal{P}_{12}\left(2+\mathcal{P}_{11} \mathcal{P}_{22}\right)
\end{array}
$$

The system is of rank four in the four index objects, a full list is given in Appendix C.

The $A_{3}$ arises from differentiation of the $A_{2}$ from $V_{4} \otimes V_{3}$ and use of the $A_{3}$ in $V_{4} \otimes V_{4}$. One derives any identity from another by use of the $e$ and $f$ operators:

$$
\begin{aligned}
& \mathcal{P}_{11} \mathcal{P}_{1122}-2 \mathcal{P}_{12} \mathcal{P}_{1112}+\mathcal{P}_{22} \mathcal{P}_{1111}=-2 \mathcal{I} \mathcal{P}_{11} \\
& \mathcal{P}_{11} \mathcal{P}_{1222}-2 \mathcal{P}_{12} \mathcal{P}_{1122}+\mathcal{P}_{22} \mathcal{P}_{1112}=-2 \mathcal{I} \mathcal{P}_{12} \\
& \mathcal{P}_{11} \mathcal{P}_{2222}-2 \mathcal{P}_{12} \mathcal{P}_{1222}+\mathcal{P}_{22} \mathcal{P}_{1122}=-2 \mathcal{I} \mathcal{P}_{22}
\end{aligned}
$$


Consequently one has the invariant relation (from $V_{3} \otimes V_{3} \otimes V_{5}$ ):

$$
\begin{aligned}
\mathcal{P}_{22}^{2} \mathcal{P}_{1111}-4 \mathcal{P}_{22} \mathcal{P}_{12} \mathcal{P}_{1112}+2\left(2 \mathcal{P}_{12}^{2}+\mathcal{P}_{11} \mathcal{P}_{22}\right) & \mathcal{P}_{1122} \\
& -4 \mathcal{P}_{11} \mathcal{P}_{12} \mathcal{P}_{1222}+\mathcal{P}_{11}^{2} \mathcal{P}_{2222}=4 \mathcal{I}
\end{aligned}
$$

The $A_{7}$ arises from straight differentiation of $\mathcal{P}_{222}$ with respect to $u_{2}$ and the lowest weight vector is,

$$
\begin{gathered}
\mathcal{P}_{22} \mathcal{P}_{2222}-3 \mathcal{P}_{222}^{2}=-12 g_{6} \mathcal{P}_{12}^{4}+30 g_{5} \mathcal{P}_{12}^{3} \mathcal{P}_{22}+9 g_{6} \mathcal{P}_{12}^{2} \mathcal{P}_{11} \mathcal{P}_{22}-30 g_{4} \mathcal{P}_{12}^{2} \mathcal{P}_{22}^{2} \\
-15 g_{5} \mathcal{P}_{12} \mathcal{P}_{11} \mathcal{P}_{22}^{2}+15 g_{3} \mathcal{P}_{12} \mathcal{P}_{22}^{3}-\frac{3}{4} g_{6} \mathcal{P}_{11}^{2} \mathcal{P}_{22}^{2}+\frac{15}{2} g_{4} \mathcal{P}_{11} \mathcal{P}_{22}^{3}-\frac{15}{4} g_{2} \mathcal{P}_{22}^{4}
\end{gathered}
$$

This system of seven equations is of rank five in the four index quantitites, so allowing the derivation of expressions for them in terms of two and three index objects, though it will be seen that this is not a very natural approach.

These identities and the consequent expressions for the $\mathcal{P}_{i j k l}$ are presented in Appendix D.

A very natural question to ask concerns the representation theoretic value of the polar form which features so prominently in the classical theory. In the new invariant formulation, this polar form is replaced by $[5,3]$

$$
\begin{aligned}
F\left(x_{1}, x_{2}\right)= & 2 g_{0}+6\left(x_{2}+x_{1}\right) g_{1}+6\left(3 x_{2} x_{1}+x_{1}^{2}+x_{2}^{2}\right) g_{2} \\
& +2\left(x_{2}+x_{1}\right)\left(x_{1}^{2}+8 x_{2} x_{1}+x_{2}^{2}\right) g_{3}+6 x_{2} x_{1}\left(3 x_{2} x_{1}+x_{1}^{2}+x_{2}^{2}\right) g_{4} \\
& +2 g_{6} x_{1}^{3} x_{2}^{3}+6 x_{1}^{2} x_{2}^{2}\left(x_{2}+x_{1}\right) g_{5},
\end{aligned}
$$

and it is straightforward to check that the combination $\mathcal{J}=\frac{1}{2} \mathcal{P}_{22}^{3} F\left(x_{1}, x_{2}\right)$ is an invariant

$$
\mathcal{J}=g_{0} \mathcal{P}_{22}^{3}-6 g_{1} \mathcal{P}_{12} \mathcal{P}_{22}^{2}+3 g_{2}\left(4 \mathcal{P}_{12}^{2}+\mathcal{P}_{11} \mathcal{P}_{22}\right) \mathcal{P}_{22}-4 g_{3} \mathcal{P}_{12}\left(2 \mathcal{P}_{12}^{2}+3 \mathcal{P}_{11} \mathcal{P}_{22}\right)
$$

$$
+3 g_{4} \mathcal{P}_{11}\left(4 \mathcal{P}_{12}^{2}+\mathcal{P}_{11} \mathcal{P}_{22}\right)-6 g_{5} \mathcal{P}_{11}^{2} \mathcal{P}_{12}+g_{6} \mathcal{P}_{11}^{3}
$$

since it lies in the kernel of both $e$ and $f$. It is the trivial representation sitting inside $G_{7} \otimes V_{3} \otimes V_{3} \otimes V_{3}$.

As a final note, the expressions for the original curve, $y_{1}^{2}=g\left(x_{1}\right)$ and $y_{2}^{2}=g\left(x_{2}\right)$ are expressible directly in terms of the two and three index quantities

$$
\begin{gathered}
\mathcal{P}_{22}\left(\mathcal{P}_{122}^{2} \mathcal{P}_{22}-\mathcal{P}_{222}^{2} \mathcal{P}_{11}\right)=\Gamma_{6}\left(\mathcal{P}_{11}, \mathcal{P}_{12}, \mathcal{P}_{22}\right) \\
\left(\mathcal{P}_{12} \mathcal{P}_{222}-\mathcal{P}_{122} \mathcal{P}_{22}\right) \mathcal{P}_{222}=\Gamma_{5}\left(\mathcal{P}_{11}, \mathcal{P}_{12}, \mathcal{P}_{22}\right)
\end{gathered}
$$

where $\Gamma_{6}$ and $\Gamma_{5}$ are homogeneous polynomials of degrees 6 and 5 , respectively, in their arguments. The first is the seven dimensional representation sitting inside $V_{4} \otimes V_{4} \otimes V_{3} \otimes V_{3}$ and the second that in $V_{4} \otimes V_{4} \otimes V_{3}$.

\section{Specialization}

It is necessary to establish a link with the classical theory in which one branch point is dismissed to infinity and where the definitions of the $\wp_{i j}$ given in the introduction stand. The relations between the $\left(x_{i}, y_{i}\right)$ and the $u_{i}$, however, is unchanged so that the expressions for derivatives are as before. The old and new symbols are easily related: $\mathcal{P}_{11}=-\mathcal{P}_{22} \wp_{12}, \mathcal{P}_{12}=-\frac{1}{2} \mathcal{P}_{22} \wp_{22}$ and $\wp_{11}=$ 
$\left(F^{0}\left(x_{1}, x_{2}\right)-4 \mathcal{I}\right) /\left(8 \mathcal{P}_{22}\right)$. Because the quadratic condition $\mathcal{P}_{12}^{2}-\mathcal{P}_{11} \mathcal{P}_{22}=1$ implies

$$
\mathcal{P}_{22}^{2}=\frac{4}{\wp_{22}^{2}+4 \wp_{12}}
$$

we obtain

$$
\mathcal{P}_{11}=-\frac{2 \wp_{12}}{\sqrt{\wp_{22}^{2}+4 \wp_{12}}}, \quad \mathcal{P}_{12}=-\frac{\wp_{22}}{\sqrt{\wp_{22}^{2}+4 \wp_{12}}}, \quad \mathcal{P}_{22}=\frac{2}{\sqrt{\wp_{22}^{2}+4 \wp_{12}}} .
$$

The verification that $\partial \wp_{22} / \partial u_{1}=\partial \wp_{12} / \partial u_{2}$ is straightforward; showing that $\partial \wp_{11} / \partial u_{2}=\partial \wp_{12} / \partial u_{1}$ rather less so.

Differentiation yields expressions for three index objects:

$$
\begin{aligned}
& \wp_{222}=\frac{\partial \wp_{22}}{\partial u_{2}}=2 \frac{\mathcal{P}_{12} \mathcal{P}_{222}-\mathcal{P}_{22} \mathcal{P}_{122}}{\mathcal{P}_{22}^{2}}=\frac{y_{1}-y_{2}}{x_{1}-x_{2}}, \\
& \wp_{122}=\frac{\partial \wp_{22}}{\partial u_{1}}=2 \frac{\mathcal{P}_{12} \mathcal{P}_{122}-\mathcal{P}_{22} \mathcal{P}_{112}}{\mathcal{P}_{22}^{2}}=\frac{x_{1} y_{2}-x_{2} y_{1}}{x_{1}-x_{2}}, \\
& \wp_{112}=\frac{\partial \wp_{12}}{\partial u_{1}}=\frac{\mathcal{P}_{11} \mathcal{P}_{122}-\mathcal{P}_{22} \mathcal{P}_{111}}{\mathcal{P}_{22}^{2}}=\frac{x_{2}^{2} y_{1}-x_{1}^{2} y_{2}}{x_{1}-x_{2}},
\end{aligned}
$$

from which follows the classical parametrization (1.11). We have also from equalities of cross differentiation (e.g. $\partial \wp_{22} / \partial u_{1}=\partial \wp_{12} / \partial u_{2}$ ), the identity

$$
-\wp_{222} \wp_{12}+\wp_{122} \wp_{22}+\wp_{112}=0 .
$$

A differentiation yields the formula for the final three index symbol,

$$
\wp_{111}=\frac{\partial \wp_{11}}{\partial u_{1}}=\frac{y_{2} \psi\left(x_{1}, x_{2}\right)-y_{1} \psi\left(x_{2}, x_{1}\right)}{4\left(x_{1}-x_{2}\right)^{2}},
$$

where [5]

$$
\begin{aligned}
\psi\left(x_{1}, x_{2}\right)= & 4 x_{1}^{3} x_{2}\left(3 x_{1}+x_{2}\right)+60 g_{4} x_{1}^{3} x_{2}+20 g_{3} x_{1}^{2}\left(x_{1}+3 x_{2}\right) \\
& +30 g_{2} x_{1}\left(x_{1}+x_{2}\right)+6 g_{1}\left(3 x_{1}+x_{2}\right)+4 g_{0} .
\end{aligned}
$$

In order to recover the classical equations for the many index $\wp$ functions, we start by differentiating equations (4.2) in order to express the $\mathcal{P}_{i j k}$ in terms of $\wp_{12}$, $\wp_{22}, \wp_{112}, \wp_{122}$ and $\wp_{222}$. Further $\mathcal{I}$ is expressed in terms of $\wp_{11}, \wp_{12}$ and $\wp_{22}$ from the relation for $\wp_{11}$ given above and the definition of $F^{0}\left(x_{1}, x_{2}\right)$. Substituting these expressions into the seven dimensional representation coming from $V_{4} \otimes V_{4}$, setting $g_{6}=0, g_{5}=2 / 3$ and solving for the binomial terms gives

$$
\wp_{222} \wp_{122}=2 \wp_{12}^{2}+15 \wp_{12} \wp_{22} g_{4}+10 \wp_{12} g_{3}+4 \wp_{22}^{2} \wp_{12}-2 \wp_{22} \wp_{11}+3 g_{1},
$$

$$
\wp_{112} \wp_{222}=2 \wp_{12}^{2} \wp_{22}+4 \wp_{11} \wp_{12}+2 \wp_{11} \wp_{22}^{2}+10 g_{3} \wp_{12} \wp_{22}+15 g_{2} \wp_{12}-3 g_{1} \wp_{22} \text {, }
$$

$$
\begin{aligned}
\wp_{112} \wp_{122} & =2 \wp_{12}^{3}+10 \wp_{12}^{2} g_{3}+2 \wp_{22} \wp_{11} \wp_{12}+3 \wp_{12} g_{1}-g_{0} \wp_{22}, \\
(4.13) \quad \wp_{112}^{2} & =4 \wp_{12}^{2} \wp_{11}+15 \wp_{12}^{2} g_{2}-6 g_{1} \wp_{12} \wp_{22}+g_{0} \wp_{22}^{2} .
\end{aligned}
$$


These are to be compared with the classical formulae of Appendix E when the $\lambda$ 's are replaced by the appropriate multiples of $g$ 's. Note that the identities involving $\wp_{111}$ are so far missing from the above. To obtain these we should use the relation (4.7), rewritten to express $\wp_{111}$ as a linear combination of $\wp_{222}$ and $\wp_{122}$, first to obtain the products $\wp_{111} \wp_{112}$ etc. in terms of what we have already then finally the square $\wp_{111}^{2}$.

Differentiating once more the expressions we obtained for the reductions of the $\mathcal{P}_{i j k}$ to give the four index objects, we may substitute into the five dimensional representation inside $V_{4} \otimes V_{4}$ and solve for $\wp_{2222}, \wp_{1222}, \wp_{1122}$ and $\wp_{1112}$

$$
\begin{aligned}
& \wp_{2222}=4 \wp_{12}+6 \wp_{22}^{2}+15 g_{4} \wp_{22}+10 g_{3}, \\
& \wp_{1222}=6 \wp_{12} \wp_{22}+15 g_{4} \wp_{12}-2 \wp_{11}, \\
& \wp_{1122}=4 \wp_{12}^{2}+10 g_{3} \wp_{12}+2 \wp_{22} \wp_{11}, \\
& \wp_{1112}=15 g_{2} \wp_{12}+6 \wp_{11} \wp_{12}-g_{0}-3 g_{1} \wp_{22} .
\end{aligned}
$$

These are to be compared with the classical equations of Appendix F, with the $g_{i}$ replaced by the corresponding $\lambda_{i}$. Again, $\wp_{1111}$ is missing from our set but it can easily be found by taking the double $u_{1}$ derivative of the $\wp_{1222}$ equation and using, say, the expression for $\wp_{1112}$.

Finally, let us return to the covariant classical equations (2.14). Recall that the indexed $\wp$-functions there do transform as representations of $S L_{2}(\mathbb{C})$. These equations can be seen to be an equality between five dimensional representations. The left hand side is built from the representations with bases $\left\{\wp_{2222}, \wp_{1222}, \wp_{1122}, \wp_{1112}\right.$, $\left.\wp_{1111}\right\}$ and $\left\{\wp_{22}^{2}, \wp_{22} \wp_{12}, 2 \wp_{12}^{2}+\wp_{11} \wp_{22}, \wp_{11} \wp_{12}, \wp_{11}^{2}\right\}$, the right hand side from the five dimensional representation sitting inside $\operatorname{Sym}\left(G_{7} \otimes G_{7}\right)$ and that in the product of $G_{7}$ and $\left\{\wp_{11}, \wp_{12}, \wp_{22}\right\}$. Application of the $e$ and $f$ operators allows one to construct the whole set of equations from any individual equation. The same would go for any identities satisfied by the classical $\wp$ functions for the generic curve and this will form the subject of a further paper [3].

\section{Conclusions}

In this paper we have presented a purely algebraic approach to the uniformization of the general genus two hyperelliptic curve in which all identities can be interpreted as covariants for the action of $S L_{2}(\mathbb{C})$. In order to be able to derive such identities we have defined new sets of covariant, indexed $\mathcal{P}$ functions for the generic curve and we have shown that the classical theory is recovered when one branch point is moved to infinity.

A number of questions remain.

The first concerns the nature of the function $\Upsilon$ which satisfies a Monge-Ampere equation. Is this function simply related to an entire function? The second concerns the general structure of the identities. Are all identities generated by a finite number of tensoring and intertwining operations?

And there are further directions for study.

Given that it is possible to apply these methods to the classically defined, covariant $\wp$ functions [3], can we use the same approach outlined here to derive identities, as opposed to merely verifying them?

In another direction, can we obtain expansions of the function $\Upsilon$ in higher and higher degree covariants analogous to the classical expansions? 
Finally, how do we extend this treatment to curves of higher genera and, further, to curves of the forms,

$$
y^{p}=\lambda_{2 g+2} x^{2 g+2}+\lambda_{2 g+1} x^{2 g+1}+\lambda_{2 g} x^{2 g}+\ldots ?
$$

\section{ACKNOWLEDGEMENTS}

All three authors gratefully acknowledge the support of the Isaac Newton Institute for the Mathematical Sciences, Cambridge, UK, during the Autumn of 2001 when this work was instigated. We would like to thank the other participants of the Integrable Systems programme and, in particular Victor Buchstaber for some stimulating conversations and Yura Brezhnev for a number of useful remarks.

Appendix A. The EXPRESSiOn FOR $\mathcal{I}^{2}$.

$$
\begin{aligned}
\mathcal{I}^{2} & =-\frac{3}{16} \mathcal{P}_{11} \mathcal{P}_{22}^{5}\left(-6 g_{1}^{2}+5 g_{2} g_{0}\right)+\frac{15}{32} \mathcal{P}_{11}^{2} \mathcal{P}_{22}^{4}\left(-16 g_{3} g_{1}+2 g_{4} g_{0}+15 g_{2}^{2}\right) \\
& +\frac{1}{16} \mathcal{P}_{11}^{3} \mathcal{P}_{22}^{3}\left(-36 g_{5} g_{1}+225 g_{4} g_{2}-200 g_{3}^{2}+g_{6} g_{0}\right) \\
& +\frac{15}{32} \mathcal{P}_{11}^{4} \mathcal{P}_{22}^{2}\left(-16 g_{5} g_{3}+2 g_{6} g_{2}+15 g_{4}^{2}\right)-\frac{3}{16} \mathcal{P}_{11}^{5} \mathcal{P}_{22}\left(5 g_{6} g_{4}-6 g_{5}^{2}\right) \\
& +\frac{15}{8} \mathcal{P}_{12} \mathcal{P}_{11} \mathcal{P}_{22}{ }^{4}\left(-3 g_{2} g_{1}+2 g_{3} g_{0}\right)-\frac{15}{8} \mathcal{P}_{12} \mathcal{P}_{11}^{2} \mathcal{P}_{22}^{3}\left(10 g_{3} g_{2}-9 g_{1} g_{4}+g_{5} g_{0}\right) \\
& +\frac{15}{8} \mathcal{P}_{12} \mathcal{P}_{11}^{3} \mathcal{P}_{22}^{2}\left(-10 g_{4} g_{3}-g_{1} g_{6}+9 g_{5} g_{2}\right)+\frac{15}{8} \mathcal{P}_{12} \mathcal{P}_{11}{ }^{4} \mathcal{P}_{22}\left(2 g_{6} g_{3}-3 g_{5} g_{4}\right) \\
& -\frac{15}{2} \mathcal{P}_{12}^{2} \mathcal{P}_{11} \mathcal{P}_{22}^{3}\left(-2 g_{3} g_{1}+g_{4} g_{0}\right)+\frac{9}{8} \mathcal{P}_{12}^{2} \mathcal{P}_{11}^{2} \mathcal{P}_{22}{ }^{2}\left(25 g_{4} g_{2}+g_{6} g_{0}-16 g_{5} g_{1}\right) \\
& -\frac{15}{2} \mathcal{P}_{12}^{2} \mathcal{P}_{11}^{3} \mathcal{P}_{22}\left(-2 g_{5} g_{3}+g_{6} g_{2}\right)+\frac{15}{2} \mathcal{P}_{12}^{3} \mathcal{P}_{11} \mathcal{P}_{22}^{2}\left(-3 g_{1} g_{4}+g_{5} g_{0}\right) \\
& -\frac{15}{2} \mathcal{P}_{12}^{3} \mathcal{P}_{11}^{2} \mathcal{P}_{22}\left(3 g_{5} g_{2}-g_{1} g_{6}\right)-3 \mathcal{P}_{12}^{4} \mathcal{P}_{11} \mathcal{P}_{22}\left(-6 g_{5} g_{1}+g_{6} g_{0}\right)+\frac{1}{32} \mathcal{P}_{11}^{6} g_{6}{ }^{2} \\
& +\frac{15}{2} \mathcal{P}_{11}^{2} \mathcal{P}_{12}^{4} g_{6} g_{2}+\frac{15}{8} \mathcal{P}_{11}^{4} \mathcal{P}_{12}^{2} g_{6} g_{4}-\frac{3}{8} \mathcal{P}_{11}^{5} \mathcal{P}_{12} g_{6} g_{5}-5 \mathcal{P}_{11}^{3} \mathcal{P}_{12}^{3} g_{6} g_{3} \\
& -6 \mathcal{P}_{11} \mathcal{P}_{12}^{5} g_{6} g_{1}+\frac{15}{8} \mathcal{P}_{12}^{2} g_{2} g_{0} \mathcal{P}_{22}^{4}-6 \mathcal{P}_{12}^{5} g_{5} g_{0} \mathcal{P}_{22}+\frac{15}{2} \mathcal{P}_{12}^{4} g_{4} g_{0} \mathcal{P}_{22}{ }^{2} \\
& -5 \mathcal{P}_{12}^{3} g_{3} g_{0} \mathcal{P}_{22}^{3}-\frac{3}{8} \mathcal{P}_{12} g_{1} g_{0} \mathcal{P}_{22}{ }^{5}+\frac{1}{32} g_{0}^{2} \mathcal{P}_{22}^{6}+2 \mathcal{P}_{12}^{6} g_{6} g_{0} \\
&
\end{aligned}
$$


Appendix B. The $A_{7}$ Representation in Quadratics in $\mathcal{P}_{i j k}$.

$$
\begin{aligned}
& \mathcal{P}_{222}^{2}-\frac{1}{2} \mathcal{I} \mathcal{P}_{22}^{3}=\frac{5}{2}\left(3 \mathcal{P}_{11} \mathcal{P}_{12} \mathcal{P}_{22}^{4}-4 \mathcal{P}_{12}^{3} \mathcal{P}_{22}^{3}\right) g_{3}+\frac{15}{8}\left(\mathcal{P}_{11}^{2} \mathcal{P}_{22}^{4}+8 \mathcal{P}_{12}^{4} \mathcal{P}_{22}^{2}-8 \mathcal{P}_{11} \mathcal{P}_{12}^{2} \mathcal{P}_{22}^{3}\right) g_{4} \\
& +\frac{3}{4}\left(20 \mathcal{P}_{11} \mathcal{P}_{12}^{3} \mathcal{P}_{22}^{2}-16 \mathcal{P}_{12}^{5} \mathcal{P}_{22}-5 \mathcal{P}_{11}^{2} \mathcal{P}_{12} \mathcal{P}_{22}^{3}\right) g_{5}+\frac{1}{8}\left(32 \mathcal{P}_{12}^{6}-\mathcal{P}_{11}^{3} \mathcal{P}_{22}^{3}-48 \mathcal{P}_{11} \mathcal{P}_{12}^{4} \mathcal{P}_{22}\right. \\
& \left.+18 \mathcal{P}_{11}^{2} \mathcal{P}_{12}^{2} \mathcal{P}_{22}^{2}\right) g_{6}+\frac{1}{8} g_{0} \mathcal{P}_{22}^{6}-\frac{3}{4} \mathcal{P}_{12} g_{1} \mathcal{P}_{22}^{5}+\frac{15}{8}\left(2 \mathcal{P}_{12}^{2} \mathcal{P}_{22}^{4}-\mathcal{P}_{11} \mathcal{P}_{22}^{5}\right) g_{2} \\
& \mathcal{P}_{122} \mathcal{P}_{222}-\frac{1}{2} \mathcal{P}_{12} \mathcal{I} \mathcal{P}_{22}^{2}=\frac{5}{2}\left(\mathcal{P}_{11}^{2} \mathcal{P}_{22}^{4}-2 \mathcal{P}_{11} \mathcal{P}_{12}^{2} \mathcal{P}_{22}^{3}\right) g_{3}+\frac{15}{8}\left(4 \mathcal{P}_{11} \mathcal{P}_{12}^{3} \mathcal{P}_{22}^{2}-3 \mathcal{P}_{11}^{2} \mathcal{P}_{12} \mathcal{P}_{22}^{3}\right) g_{4} \\
& +\frac{3}{4}\left(8 \mathcal{P}_{11}^{2} \mathcal{P}_{12}^{2} \mathcal{P}_{22}^{2}-8 \mathcal{P}_{11} \mathcal{P}_{12}^{4} \mathcal{P}_{22}-\mathcal{P}_{11}^{3} \mathcal{P}_{22}^{3}\right) g_{5}+\frac{1}{8}\left(5 \mathcal{P}_{12} \mathcal{P}_{11}^{3} \mathcal{P}_{22}^{2}-20 \mathcal{P}_{11}^{2} \mathcal{P}_{12}^{3} \mathcal{P}_{22}\right. \\
& \left.+16 \mathcal{P}_{11} \mathcal{P}_{12}^{5}\right) g_{6}-\frac{3}{4} \mathcal{P}_{11} g_{1} \mathcal{P}_{22}^{5}+\frac{1}{8} g_{0} \mathcal{P}_{12} \mathcal{P}_{22}^{5}+\frac{15}{8} \mathcal{P}_{12} \mathcal{P}_{11} g_{2} \mathcal{P}_{22}^{4} \\
& 3 \mathcal{P}_{122}^{2}+2 \mathcal{P}_{112} \mathcal{P}_{222}-\frac{1}{2}\left(4 \mathcal{P}_{12}^{2} \mathcal{P}_{22}+\mathcal{P}_{11} \mathcal{P}_{22}^{2}\right) \mathcal{I}=-\frac{25}{2} \mathcal{P}_{12} \mathcal{P}_{11}^{2} g_{3} \mathcal{P}_{22}^{3}+\frac{75}{8}\left(2 \mathcal{P}_{11}^{2} \mathcal{P}_{12}^{2} \mathcal{P}_{22}^{2}\right. \\
& \left.-\mathcal{P}_{11}^{3} \mathcal{P}_{22}^{3}\right) g_{4}+\frac{15}{4}\left(3 \mathcal{P}_{12} \mathcal{P}_{11}^{3} \mathcal{P}_{22}^{2}-4 \mathcal{P}_{11}^{2} \mathcal{P}_{12}^{3} \mathcal{P}_{22}\right) g_{5}+\frac{5}{8}\left(8 \mathcal{P}_{11}^{2} \mathcal{P}_{12}^{4}-8 \mathcal{P}_{12}^{2} \mathcal{P}_{11}^{3} \mathcal{P}_{22}\right. \\
& \left.+\mathcal{P}_{11}^{4} \mathcal{P}_{22}^{2}\right) g_{6}+\frac{5}{8}\left(2 \mathcal{P}_{12}^{2} \mathcal{P}_{22}^{4}-\mathcal{P}_{11} \mathcal{P}_{22}^{5}\right) g_{0}-\frac{15}{4} g_{1} \mathcal{P}_{11} \mathcal{P}_{12} \mathcal{P}_{22}^{4}+\frac{75}{8} \mathcal{P}_{11}^{2} g_{2} \mathcal{P}_{22}^{4} \\
& 9 \mathcal{P}_{122} \mathcal{P}_{112}+\mathcal{P}_{111} \mathcal{P}_{222}-\left(3 \mathcal{P}_{12} \mathcal{P}_{11} \mathcal{P}_{22}+2 \mathcal{P}_{12}^{3}\right) \mathcal{I}=-25 \mathcal{P}_{11}^{3} g_{3} \mathcal{P}_{22}^{3}+\frac{75}{4} \mathcal{P}_{12} \mathcal{P}_{11}^{3} g_{4} \mathcal{P}_{22}^{2} \\
& +\frac{15}{2}\left(\mathcal{P}_{11}^{4} \mathcal{P}_{22}^{2}-2 \mathcal{P}_{12}^{2} \mathcal{P}_{11}^{3} \mathcal{P}_{22}\right) g_{5}+\frac{5}{4}\left(4 \mathcal{P}_{12}^{3} \mathcal{P}_{11}^{3}-3 \mathcal{P}_{12} \mathcal{P}_{11}^{4} \mathcal{P}_{22}\right) g_{6}+\frac{5}{4}\left(4 \mathcal{P}_{12}^{3} \mathcal{P}_{22}^{3}\right. \\
& \left.-3 \mathcal{P}_{11} \mathcal{P}_{12} \mathcal{P}_{22}^{4}\right) g_{0}+\frac{15}{2}\left(\mathcal{P}_{11}^{2} \mathcal{P}_{22}^{4}-2 \mathcal{P}_{11} \mathcal{P}_{12}^{2} \mathcal{P}_{22}^{3}\right) g_{1}+\frac{75}{4} g_{2} \mathcal{P}_{11}^{2} \mathcal{P}_{12} \mathcal{P}_{22}^{3} \\
& 2 \mathcal{P}_{122} \mathcal{P}_{111}+3 \mathcal{P}_{112}^{2}-\frac{1}{2}\left(\mathcal{P}_{11}^{2} \mathcal{P}_{22}+4 \mathcal{P}_{12}^{2} \mathcal{P}_{11}\right) \mathcal{I}=-\frac{25}{2} g_{3} \mathcal{P}_{12} \mathcal{P}_{11}^{3} \mathcal{P}_{22}^{2}+\frac{75}{8} \mathcal{P}_{11}^{4} g_{4} \mathcal{P}_{22}^{2} \\
& -\frac{15}{4} \mathcal{P}_{12} \mathcal{P}_{11}^{4} g_{5} \mathcal{P}_{22}+\frac{15}{8}\left(2 \mathcal{P}_{12}^{2} \mathcal{P}_{11}^{4}-\mathcal{P}_{11}^{5} \mathcal{P}_{22}\right) g_{6}+\frac{5}{8}\left(\mathcal{P}_{11}^{2} \mathcal{P}_{22}^{4}+8 \mathcal{P}_{12}^{4} \mathcal{P}_{22}^{2}-8 \mathcal{P}_{11} \mathcal{P}_{12}^{2} \mathcal{P}_{22}^{3}\right) g_{0} \\
& +\frac{15}{4}\left(3 \mathcal{P}_{11}^{2} \mathcal{P}_{12} \mathcal{P}_{22}^{3}-4 \mathcal{P}_{11} \mathcal{P}_{12}^{3} \mathcal{P}_{22}^{2}\right) g_{1}+\frac{75}{8}\left(2 \mathcal{P}_{11}^{2} \mathcal{P}_{12}^{2} \mathcal{P}_{22}^{2}-\mathcal{P}_{11}^{3} \mathcal{P}_{22}^{3}\right) g_{2} \\
& \mathcal{P}_{112} \mathcal{P}_{111}-\frac{1}{2} \mathcal{P}_{12} \mathcal{P}_{11}^{2} \mathcal{I}=\frac{5}{2}\left(\mathcal{P}_{11}^{4} \mathcal{P}_{22}^{2}-2 \mathcal{P}_{12}^{2} \mathcal{P}_{11}^{3} \mathcal{P}_{22}\right) g_{3}+\frac{15}{8} g_{4} \mathcal{P}_{12} \mathcal{P}_{11}^{4} \mathcal{P}_{22}-\frac{3}{4} \mathcal{P}_{11}^{5} g_{5} \mathcal{P}_{22} \\
& +\frac{1}{8}\left(16 \mathcal{P}_{12}^{5} \mathcal{P}_{22}-20 \mathcal{P}_{11} \mathcal{P}_{12}^{3} \mathcal{P}_{22}^{2}+5 \mathcal{P}_{11}^{2} \mathcal{P}_{12} \mathcal{P}_{22}^{3}\right) g_{0}+\frac{3}{4}\left(8 \mathcal{P}_{11}^{2} \mathcal{P}_{12}^{2} \mathcal{P}_{22}^{2}-8 \mathcal{P}_{11} \mathcal{P}_{12}^{4} \mathcal{P}_{22}\right. \\
& \left.-\mathcal{P}_{11}^{3} \mathcal{P}_{22}^{3}\right) g_{1}+\frac{15}{8}\left(3 \mathcal{P}_{11}^{2} \mathcal{P}_{12}^{3} \mathcal{P}_{22}-4 \mathcal{P}_{12} \mathcal{P}_{11}^{3} \mathcal{P}_{22}^{2}\right) g_{2}+\frac{1}{8} \mathcal{P}_{12} \mathcal{P}_{11}^{5} g_{6} \\
& \mathcal{P}_{111}^{2}-\frac{1}{2} \mathcal{P}_{11}^{3} \mathcal{I}=\frac{5}{2}\left(3 \mathcal{P}_{12} \mathcal{P}_{11}^{4} \mathcal{P}_{22}-4 \mathcal{P}_{12}^{3} \mathcal{P}_{11}^{3}\right) g_{3}+\frac{15}{8}\left(2 \mathcal{P}_{12}^{2} \mathcal{P}_{11}^{4}-\mathcal{P}_{11}^{5} \mathcal{P}_{22}\right) g_{4}-\frac{3}{4} g_{5} \mathcal{P}_{12} \mathcal{P}_{11}^{5} \\
& +\frac{1}{8} \mathcal{P}_{11}^{6} g_{6}+\frac{1}{8}\left(32 \mathcal{P}_{12}^{6}-\mathcal{P}_{11}^{3} \mathcal{P}_{22}^{3}-48 \mathcal{P}_{11} \mathcal{P}_{12}^{4} \mathcal{P}_{22}+18 \mathcal{P}_{11}^{2} \mathcal{P}_{12}^{2} \mathcal{P}_{22}^{2}\right) g_{0}+\frac{3}{4}\left(20 \mathcal{P}_{11}^{2} \mathcal{P}_{12}^{3} \mathcal{P}_{22}\right. \\
& \left.-5 \mathcal{P}_{12} \mathcal{P}_{11}^{3} \mathcal{P}_{22}^{2}-16 \mathcal{P}_{11} \mathcal{P}_{12}^{5}\right) g_{1}+\frac{15}{8}\left(8 \mathcal{P}_{11}^{2} \mathcal{P}_{12}^{4}-8 \mathcal{P}_{12}^{2} \mathcal{P}_{11}^{3} \mathcal{P}_{22}+\mathcal{P}_{11}^{4} \mathcal{P}_{22}^{2}\right) g_{2}
\end{aligned}
$$


Appendix C. The $A_{5}$ Inside $V_{5} \otimes V_{3}$.

$$
\begin{aligned}
& \mathcal{P}_{2222} \mathcal{P}_{12}-\mathcal{P}_{1222} \mathcal{P}_{22}=-\frac{3}{2} g_{1} \mathcal{P}_{22}{ }^{4}-15 \mathcal{P}_{12}{ }^{2} g_{3} \mathcal{P}_{22}{ }^{2}+15 \mathcal{P}_{12}{ }^{3} g_{4} \mathcal{P}_{22}-6 \mathcal{P}_{12}{ }^{4} g_{5} \\
& +\frac{15}{2} \mathcal{P}_{12} g_{2} \mathcal{P}_{22}{ }^{3}-3 \mathcal{P}_{11} \mathcal{P}_{12}{ }^{2} g_{5} \mathcal{P}_{22}+3 \mathcal{P}_{11} \mathcal{P}_{12}{ }^{3} g_{6}+\frac{3}{2} \mathcal{P}_{11}{ }^{2} g_{5} \mathcal{P}_{22}{ }^{2}-\frac{3}{2} \mathcal{P}_{11}{ }^{2} \mathcal{P}_{12} g_{6} \mathcal{P}_{22} \\
& 2 \mathcal{P}_{12} \mathcal{P}_{1222}+\mathcal{P}_{11} \mathcal{P}_{2222}-3 \mathcal{P}_{1122} \mathcal{P}_{22}=3 g_{1} \mathcal{P}_{12} \mathcal{P}_{22}{ }^{3}-12 \mathcal{P}_{11} \mathcal{P}_{12}{ }^{3} g_{5} \\
& -9 \mathcal{P}_{12} \mathcal{P}_{11}{ }^{2} g_{5} \mathcal{P}_{22}+6 \mathcal{P}_{11}{ }^{2} \mathcal{P}_{12}{ }^{2} g_{6}-30 \mathcal{P}_{11} \mathcal{P}_{12} g_{3} \mathcal{P}_{22}{ }^{2}+30 \mathcal{P}_{11} \mathcal{P}_{12}{ }^{2} g_{4} \mathcal{P}_{22} \\
& +\frac{15}{2} \mathcal{P}_{11} g_{2} \mathcal{P}_{22}{ }^{3}-\frac{3}{2} \mathcal{P}_{11}{ }^{3} g_{6} \mathcal{P}_{22}-\frac{3}{2} g_{0} \mathcal{P}_{22}{ }^{4}+\frac{15}{2} g_{4} \mathcal{P}_{11}{ }^{2} \mathcal{P}_{22}{ }^{2} \\
& 3 \mathcal{P}_{11} \mathcal{P}_{1222}-3 \mathcal{P}_{1112} \mathcal{P}_{22}=9 g_{1} \mathcal{P}_{12}{ }^{2} \mathcal{P}_{22}{ }^{2}-9 \mathcal{P}_{12}{ }^{2} \mathcal{P}_{11}{ }^{2} g_{5}-\frac{45}{2} g_{2} \mathcal{P}_{11} \mathcal{P}_{12} \mathcal{P}_{22}{ }^{2} \\
& +\frac{9}{2} \mathcal{P}_{12} \mathcal{P}_{11}{ }^{3} g_{6}-\frac{9}{2} g_{0} \mathcal{P}_{12} \mathcal{P}_{22}{ }^{3}+\frac{45}{2} \mathcal{P}_{12} g_{4} \mathcal{P}_{11}{ }^{2} \mathcal{P}_{22}+9 g_{1} \mathcal{P}_{11} \mathcal{P}_{22}{ }^{3}-9 \mathcal{P}_{11}{ }^{3} g_{5} \mathcal{P}_{22} \\
& 3 \mathcal{P}_{1122} \mathcal{P}_{11}-2 \mathcal{P}_{12} \mathcal{P}_{1112}-\mathcal{P}_{1111} \mathcal{P}_{22}=12 g_{1} \mathcal{P}_{12}{ }^{3} \mathcal{P}_{22}-30 g_{2} \mathcal{P}_{11} \mathcal{P}_{12}{ }^{2} \mathcal{P}_{22} \\
& -6 g_{0} \mathcal{P}_{12}{ }^{2} \mathcal{P}_{22}{ }^{2}+9 g_{1} \mathcal{P}_{11} \mathcal{P}_{12} \mathcal{P}_{22}{ }^{2}-3 g_{5} \mathcal{P}_{12} \mathcal{P}_{11}{ }^{3}-\frac{15}{2} \mathcal{P}_{11}{ }^{2} g_{2} \mathcal{P}_{22}{ }^{2} \\
& +\frac{3}{2} \mathcal{P}_{11}{ }^{4} g_{6}+\frac{3}{2} \mathcal{P}_{11} g_{0} \mathcal{P}_{22}{ }^{3}-\frac{15}{2} g_{4} \mathcal{P}_{11}{ }^{3} \mathcal{P}_{22}+30 g_{3} \mathcal{P}_{11}{ }^{2} \mathcal{P}_{12} \mathcal{P}_{22} \\
& \mathcal{P}_{11} \mathcal{P}_{1112}-\mathcal{P}_{12} \mathcal{P}_{1111}=6 g_{1} \mathcal{P}_{12}{ }^{4}-15 g_{2} \mathcal{P}_{11} \mathcal{P}_{12}{ }^{3}-3 g_{0} \mathcal{P}_{12}{ }^{3} \mathcal{P}_{22}+3 g_{1} \mathcal{P}_{11} \mathcal{P}_{12}{ }^{2} \mathcal{P}_{22} \\
& +\frac{3}{2} \mathcal{P}_{12} \mathcal{P}_{11} g_{0} \mathcal{P}_{22}{ }^{2}-\frac{15}{2} g_{4} \mathcal{P}_{12} \mathcal{P}_{11}{ }^{3}+15 g_{3} \mathcal{P}_{11}{ }^{2} \mathcal{P}_{12}{ }^{2}-\frac{3}{2} g_{1} \mathcal{P}_{11}{ }^{2} \mathcal{P}_{22}{ }^{2}+\frac{3}{2} g_{5} \mathcal{P}_{11}{ }^{4}
\end{aligned}
$$

Appendix D. The $A_{7}$ Inside $V_{5} \otimes V_{3}$.

$$
\begin{aligned}
& \mathcal{P}_{2222} \mathcal{P}_{22}-\mathcal{P}_{222}{ }^{2}=\frac{15}{4} g_{2} \mathcal{P}_{22}{ }^{4}-15 \mathcal{P}_{12} g_{3} \mathcal{P}_{22}{ }^{3}+30 \mathcal{P}_{12}{ }^{2} g_{4} \mathcal{P}_{22}{ }^{2} \\
&-30 \mathcal{P}_{12}{ }^{3} g_{5} \mathcal{P}_{22}+12 \mathcal{P}_{12}{ }^{4} g_{6}-\frac{15}{2} \mathcal{P}_{11} g_{4} \mathcal{P}_{22}{ }^{3}+15 \mathcal{P}_{11} \mathcal{P}_{12} g_{5} \mathcal{P}_{22}{ }^{2} \\
&-9 \mathcal{P}_{11} \mathcal{P}_{12}{ }^{2} g_{6} \mathcal{P}_{22}+\frac{3}{4} \mathcal{P}_{11}{ }^{2} g_{6} \mathcal{P}_{22}{ }^{2} . \\
& \mathcal{P}_{12} \mathcal{P}_{2222}-3 \mathcal{P}_{122} \mathcal{P}_{222}+2 \mathcal{P}_{1222} \mathcal{P}_{22}=-\frac{15}{2} g_{2} \mathcal{P}_{12} \mathcal{P}_{22}{ }^{3}+15 \mathcal{P}_{12}{ }^{2} g_{3} \mathcal{P}_{22}{ }^{2} \\
& \quad-15 \mathcal{P}_{12}{ }^{3} g_{4} \mathcal{P}_{22}+6 \mathcal{P}_{12}{ }^{4} g_{5}+45 \mathcal{P}_{12} \mathcal{P}_{11} g_{4} \mathcal{P}_{22}{ }^{2}-42 \mathcal{P}_{11} \mathcal{P}_{12}{ }^{2} g_{5} \mathcal{P}_{22}+ \\
&+15 \mathcal{P}_{11} \mathcal{P}_{12}{ }^{3} g_{6}-\frac{15}{2} \mathcal{P}_{12} \mathcal{P}_{11}{ }^{2} g_{6} \mathcal{P}_{22}-\frac{45}{2} \mathcal{P}_{11} g_{3} \mathcal{P}_{22}{ }^{3}+\frac{39}{4} \mathcal{P}_{11}{ }^{2} g_{5} \mathcal{P}_{22}{ }^{2} \\
&+\frac{15}{4} g_{1} \mathcal{P}_{22}{ }^{4} .
\end{aligned}
$$




$$
\begin{aligned}
& 8 \mathcal{P}_{12} \mathcal{P}_{1222}+\mathcal{P}_{11} \mathcal{P}_{2222}-9 \mathcal{P}_{122}{ }^{2}-6 \mathcal{P}_{112} \mathcal{P}_{222}+6 \mathcal{P}_{1122} \mathcal{P}_{22}=75 \mathcal{P}_{12} \mathcal{P}_{11} g_{3} \mathcal{P}_{22}{ }^{2} \\
& -90 \mathcal{P}_{12} \mathcal{P}_{11}{ }^{2} g_{5} \mathcal{P}_{22}+30 \mathcal{P}_{11} \mathcal{P}_{12}{ }^{3} g_{5}+30 \mathcal{P}_{12}{ }^{2} \mathcal{P}_{11}{ }^{2} g_{6}+\frac{375}{4} \mathcal{P}_{11}{ }^{2} g_{4} \mathcal{P}_{22}{ }^{2} \\
& -\frac{15}{2} \mathcal{P}_{11}{ }^{3} g_{6} \mathcal{P}_{22}-75 \mathcal{P}_{11} g_{2} \mathcal{P}_{22}{ }^{3}+\frac{15}{4} g_{0} \mathcal{P}_{22}{ }^{4}+15 g_{1} \mathcal{P}_{12} \mathcal{P}_{22}{ }^{3}-75 \mathcal{P}_{12}{ }^{2} \mathcal{P}_{11} g_{4} \mathcal{P}_{22} \\
& 6 \mathcal{P}_{12} \mathcal{P}_{1122}+2 \mathcal{P}_{11} \mathcal{P}_{1222}-9 \mathcal{P}_{122} \mathcal{P}_{112}-\mathcal{P}_{111} \mathcal{P}_{222}+2 \mathcal{P}_{1112} \mathcal{P}_{22}=15 \mathcal{P}_{12}{ }^{2} \mathcal{P}_{11}{ }^{2} g_{5} \\
& -\frac{75}{2} \mathcal{P}_{12} \mathcal{P}_{11}{ }^{2} g_{4} \mathcal{P}_{22}+\frac{15}{2} \mathcal{P}_{12} \mathcal{P}_{11}{ }^{3} g_{6}-\frac{75}{2} g_{2} \mathcal{P}_{11} \mathcal{P}_{12} \mathcal{P}_{22}{ }^{2}+\frac{15}{2} g_{0} \mathcal{P}_{12} \mathcal{P}_{22}{ }^{3} \\
& +15 g_{1} \mathcal{P}_{12}{ }^{2} \mathcal{P}_{22}{ }^{2}+75 \mathcal{P}_{11}{ }^{2} g_{3} \mathcal{P}_{22}{ }^{2}-\frac{45}{2} \mathcal{P}_{11}{ }^{3} g_{5} \mathcal{P}_{22}-\frac{45}{2} g_{1} \mathcal{P}_{11} \mathcal{P}_{22}{ }^{3} \\
& 8 \mathcal{P}_{12} \mathcal{P}_{1112}+6 \mathcal{P}_{11} \mathcal{P}_{1122}-6 \mathcal{P}_{122} \mathcal{P}_{111}-9 \mathcal{P}_{112}{ }^{2}+\mathcal{P}_{1111} \mathcal{P}_{22}=-75 g_{2} \mathcal{P}_{11} \mathcal{P}_{12}{ }^{2} \mathcal{P}_{22} \\
& +30 g_{0} \mathcal{P}_{12}{ }^{2} \mathcal{P}_{22}{ }^{2}+30 g_{1} \mathcal{P}_{12}{ }^{3} \mathcal{P}_{22}+75 g_{3} \mathcal{P}_{12} \mathcal{P}_{11}{ }^{2} \mathcal{P}_{22}+15 g_{5} \mathcal{P}_{12} \mathcal{P}_{11}{ }^{3} \\
& -90 g_{1} \mathcal{P}_{11} \mathcal{P}_{12} \mathcal{P}_{22}{ }^{2}-75 \mathcal{P}_{11}{ }^{3} g_{4} \mathcal{P}_{22}+\frac{15}{4} \mathcal{P}_{11}{ }^{4} g_{6}+\frac{375}{4} g_{2} \mathcal{P}_{11}{ }^{2} \mathcal{P}_{22}{ }^{2}-\frac{15}{2} \mathcal{P}_{11} g_{0} \mathcal{P}_{22}{ }^{3} . \\
& \mathcal{P}_{12} \mathcal{P}_{1111}+2 \mathcal{P}_{11} \mathcal{P}_{1112}-3 \mathcal{P}_{112} \mathcal{P}_{111}=-15 g_{2} \mathcal{P}_{11} \mathcal{P}_{12}{ }^{3}+15 g_{0} \mathcal{P}_{12}{ }^{3} \mathcal{P}_{22}+6 g_{1} \mathcal{P}_{12}{ }^{4} \\
& +15 g_{3} \mathcal{P}_{12}{ }^{2} \mathcal{P}_{11}{ }^{2}-42 g_{1} \mathcal{P}_{11} \mathcal{P}_{12}{ }^{2} \mathcal{P}_{22}-\frac{15}{2} g_{4} \mathcal{P}_{12} \mathcal{P}_{11}{ }^{3}+45 g_{2} \mathcal{P}_{12} \mathcal{P}_{11}{ }^{2} \mathcal{P}_{22} \\
& -\frac{15}{2} \mathcal{P}_{12} \mathcal{P}_{11} g_{0} \mathcal{P}_{22}{ }^{2}-\frac{45}{2} g_{3} \mathcal{P}_{11}{ }^{3} \mathcal{P}_{22}+\frac{15}{4} g_{5} \mathcal{P}_{11}{ }^{4}+\frac{39}{4} g_{1} \mathcal{P}_{11}{ }^{2} \mathcal{P}_{22}{ }^{2} \\
& \mathcal{P}_{11} \mathcal{P}_{1111}-\mathcal{P}_{111}{ }^{2}=12 g_{0} \mathcal{P}_{12}{ }^{4}-30 g_{1} \mathcal{P}_{11} \mathcal{P}_{12}{ }^{3}+30 g_{2} \mathcal{P}_{12}{ }^{2} \mathcal{P}_{11}{ }^{2}-9 \mathcal{P}_{12}{ }^{2} \mathcal{P}_{11} g_{0} \mathcal{P}_{22} \\
& -15 g_{3} \mathcal{P}_{12} \mathcal{P}_{11}{ }^{3}+15 \mathcal{P}_{12} g_{1} \mathcal{P}_{11}{ }^{2} \mathcal{P}_{22}+\frac{15}{4} g_{4} \mathcal{P}_{11}{ }^{4}-\frac{15}{2} g_{2} \mathcal{P}_{11}{ }^{3} \mathcal{P}_{22}+\frac{3}{4} \mathcal{P}_{11}{ }^{2} g_{0} \mathcal{P}_{22}{ }^{2}
\end{aligned}
$$

Appendix E. Classical QUadratic identities in $\wp_{i j k}$

$$
\begin{aligned}
\wp_{111}^{2} & =\frac{1}{16} \lambda_{4} \lambda_{1}^{2}+\frac{1}{4} \wp_{22} \lambda_{1}^{2}+\frac{1}{16} \lambda_{3}^{2} \lambda_{0}+4 \wp_{11}^{3}+\wp_{11}^{2} \lambda_{2}+\frac{1}{4} \wp_{11} \lambda_{3} \lambda_{1}+ \\
& +\wp_{11} \wp_{12} \lambda_{1}-\lambda_{4} \wp_{11} \lambda_{0}-\frac{1}{4} \lambda_{4} \lambda_{2} \lambda_{0}-4 \wp_{22} \wp_{11} \lambda_{0}-\wp_{22} \lambda_{2} \lambda_{0}+\frac{1}{2} \lambda_{3} \wp_{12} \lambda_{0}+\wp_{12}^{2} \lambda_{0} . \\
\wp_{112}^{2} & =\wp_{22}^{2} \lambda_{0}-\wp_{12} \wp_{22} \lambda_{1}+\wp_{12}^{2} \lambda_{2}+4 \wp_{12}^{2} \wp_{11} \\
\wp_{122}^{2} & =4 \wp_{12}^{2} \wp_{22}+\wp_{12}^{2} \lambda_{4}-4 \wp_{12} \wp_{11}+\lambda_{0} . \\
\wp_{222}^{2} & =\wp_{22} \lambda_{3}+\lambda_{4} \wp_{22}^{2}+4 \wp_{22}^{3}+4 \wp_{12} \wp_{22}+\lambda_{2}+4 \wp_{11} .
\end{aligned}
$$

$$
\begin{aligned}
\wp_{111} \wp_{112} & =-\frac{1}{2} \lambda_{2} \lambda_{0}-2 \wp_{11} \lambda_{0}+\frac{1}{8} \lambda_{1}^{2}+\frac{1}{2} \wp_{12}^{2} \lambda_{1}+\wp_{11} \wp_{12} \lambda_{2}-\frac{1}{2} \wp_{11} \wp_{22} \lambda_{1}+ \\
& +\frac{1}{8} \lambda_{3} \wp_{12} \lambda_{1}-\frac{1}{4} \lambda_{3} \wp_{22} \lambda_{0}-\wp_{12} \wp_{22} \lambda_{0}+4 \wp_{12} \wp_{11}^{2} . \\
\wp_{122} \wp_{111} & =-\frac{1}{2} \wp_{11} \lambda_{1}-\wp_{12} \lambda_{0}+2 \wp_{12}^{2} \wp_{11}+\wp_{12} \wp_{22} \lambda_{1}-\frac{1}{4} \lambda_{3} \lambda_{0}+\frac{1}{2} \wp_{11} \wp_{12} \lambda_{3}+ \\
& +\frac{1}{4} \lambda_{4} \wp_{12} \lambda_{1}+2 \wp_{22} \wp_{11}^{2}-\frac{1}{2} \lambda_{4} \wp_{22} \lambda_{0}-2 \wp_{22}^{2} \lambda_{0} .
\end{aligned}
$$




$$
\begin{aligned}
\wp_{222} \wp_{111} & =-\wp_{11} \lambda_{2}-\frac{1}{2} \wp_{12} \lambda_{1}-\wp_{12}^{2} \lambda_{3}-\frac{1}{8} \lambda_{3} \lambda_{1}-\frac{1}{8} \wp_{12} \lambda_{3}^{2}-\wp_{22}^{2} \lambda_{1}- \\
& -2 \wp_{12}^{3}-4 \wp_{11}^{2}-\frac{1}{2} \wp_{11} \wp_{22} \lambda_{3}+6 \wp_{12} \wp_{22} \wp_{11}-\frac{1}{4} \lambda_{4} \wp_{22} \lambda_{1}+ \\
& +2 \wp_{22} \wp_{12} \lambda_{2}+\frac{1}{2} \lambda_{4} \wp_{12} \lambda_{2}+2 \lambda_{4} \wp_{12} \wp_{11} . \\
\wp_{122} \wp_{112} & =-\wp_{22} \lambda_{0}+2 \wp_{12} \wp_{22} \wp_{11}+\frac{1}{2} \wp_{12} \lambda_{1}+\frac{1}{2} \wp_{12}^{2} \lambda_{3}+2 \wp_{12}^{3} . \\
\wp_{222} \wp_{112} & =\frac{1}{2} \wp_{12} \wp_{22} \lambda_{3}-\frac{1}{2} \wp_{22} \lambda_{1}+2 \wp_{12}^{2} \wp_{22}+2 \wp_{22}^{2} \wp_{11}+4 \wp_{12} \wp_{11}+\wp_{12} \lambda_{2} \\
\wp_{222} \wp_{122} & =\wp_{22} \wp_{12} \lambda_{4}+2 \wp_{12}^{2}+4 \wp_{12} \wp_{22}^{2}+\frac{1}{2} \lambda_{1}+\frac{1}{2} \wp_{12} \lambda_{3}-2 \wp_{22} \wp_{11} .
\end{aligned}
$$

Appendix F. Classical identities FOR $\wp_{i j k l}$.

$$
\begin{aligned}
& \wp_{1111}=\frac{1}{8} \lambda_{3} \lambda_{1}+\wp_{12} \lambda_{1}-3 \wp_{22} \lambda_{0}-\frac{1}{2} \lambda_{4} \lambda_{0}+6 \wp_{11}^{2}+\wp_{11} \lambda_{2} . \\
& \wp_{1112}=6 \wp_{12} \wp_{11}-\lambda_{0}-\frac{1}{2} \wp_{22} \lambda_{1}+\wp_{12} \lambda_{2} . \\
& \wp_{1122}=2 \wp_{22} \wp_{11}+4 \wp_{12}^{2}+\frac{1}{2} \wp_{12} \lambda_{3} . \\
& \wp_{1222}=\wp_{12} \lambda_{4}+6 \wp_{12} \wp_{22}-2 \wp_{11} . \\
& \wp_{2222}=6 \wp_{22}^{2}+\wp_{22} \lambda_{4}+4 \wp_{12}+\frac{1}{2} \lambda_{3} .
\end{aligned}
$$

\section{REFERENCES}

[1] Chris Athorne. Algebraic invariants and generalized Hirota derivatives. Phys. Lett. A, 256:2024,1999 .

[2] Chris Athorne. Hirota derivatives and representation theory. Glasgow Math. J., 43A:1-8, 2001.

[3] Chris Athorne, J. C. Eilbeck, and V. Z. Enolskii. Identities for classical $\wp$ functions. in preparation, 2002.

[4] H. F. Baker. Abels theorem and the allied theory including the theory of theta functions. Cambridge Univ. Press, Cambridge, 1897, reprinted 1995.

[5] H. F. Baker. Multiply Periodic Functions. Cambridge Univ. Press, Cambridge, 1907.

[6] V. M. Buchstaber, V. Z. Enolskii, and D. V. Leykin. Hyperelliptic Kleinian functions and applications. In V. M. Buchstaber and S. P. Novikov, editors, Solitons, Geometry and Topology: On the Crossroad, pages 1-34. Advances in Math. Sciences, AMS Translations, Series 2, Vol. 179, Moscow State University and University of Maryland, College Park, 1997.

[7] J. C. Eilbeck and V. Z. Enolskii. Bilinear operators and the power series for the Weierstrass $\sigma$-function. J. Phys. A: Math. Gen., 33:791-794, 2000.

[8] C. L. Siegel. Topics in complex function theory, Vol. I Elliptic functions and uniformization theory. Willey-Interscience, New York, 1969.

Department of Mathematics, University of Glasgow, Glasgow G12 8QW, UK E-mail address: c.athorne@maths.gla.ac.uk

Department of Mathematics, Heriot-Watt University, Edinburgh EH14 4AS, UK

E-mail address: J.C.Eilbeck@hw.ac.uk

Dipartimento di Fisica "E. R. Caianiello", Universita di Salerno, Via S. Allende, 84081 Baronissi (SA), ITALY

E-mail address: vze@ma.hw.ac.uk 